\title{
ESTUDO HISTÓRICO - ORGANIZACIONAL DA COMISSÃO DE CONTROLE DE INFECÇÃO HOSPITALAR DE UM HOSPITAL UNIVERSITÁRIO*
}

\author{
HISTORICAL-ORGANIZATIONAL STUDY ABOUTTHE HOSPITALINFECTION \\ CONTROL COMMITTEE OF AN UNIVERSITY HOSPITAL
}

\author{
Magda Fabbri Isaac Silvaํㄹ Branca Maria de Oliveira Santos²
}

\begin{abstract}
${ }^{1}$ Enfermeira, mestre em enfermagem, doutoranda do Programa de Pós-Graduação Interunidades da EERP-USP, Enfermeira da Comissão de Controle de Infecção Hospitalar do Hospital das Clínicas da Faculdade de Medicina de Ribeirão Preto-USP - 2 Professora Associada junto ao Departamento de Enfermagem Geral e Especializada da EERP-USP.

CorRespondêncIAs: Magda Fabbri Isaac Silva - Unidade de Emergência - Hospital das Clínicas da Faculdade de Medicina de Ribeirão Preto - USP : Comissão de Controle de Infecção Hospitalar - Rua Bernardino de Campus no 1000 - CEP 14.015-130, Ribeirão Preto SP, Fone (0XX16) 602-1168.
\end{abstract}

SILVA MFI \& OLIVEIRA SANTOS BM. Estudo histórico-organizacional da comissão de controle de infecção hospitalar de um hospital universitário. Medicina, Ribeirão Preto 34: 170-176, abr./jun. 2001.

RESUMO: Este trabalho caracteriza-se como um estudo histórico-organizacional da trajetória da Comissão de Controle de Infecção Hospitalar do Hospital das Clínicas da Faculdade de Medicina de Ribeirão Preto da Universidade de São Paulo, desde a sua constituição (1973) até o momento atual (1997), com base em atas e processos organizados cronologicamente. Para esta abordagem foi utilizada a metodologia de estudo de caso, que se caracterizou pelo exame detalhado da documentação, com vistas à determinação de categorias e subcategorias temáticas que representassem o complexo das atividades desenvolvidas pela referida comissão. As atividades desenvolvidas estiveram basicamente, centradas nas de vigilância epidemiológica das infecções, ensino e educação continuada, assessoria em projetos relacionados à planta física e segurança do trabalho. O estudo poderá contribuir como fonte de informações de fácil acesso para a própria comissão, para a constituição de novas comissões e, até mesmo, para o desenvolvimento de futuras pesquisas.

UNITERMOS: Comissões. Controle de Infecções. Infecção Hospitalar. História

\section{INTRODUÇÃO}

Pela abordagem histórica apresentada por diversos autores, a década de 50 foi caracterizada pelo início de uma nova era para as infecções hospitalares (IH) e epidemiologia ${ }^{(1 / 5)}$.Infecções hospitalares são definidas como aquelas adquiridas após a admissão do paciente ao hospital, que se manifestam durante a internação ou após a alta e podem ser relacionadas com a internação ou procedimentos hospitalares ${ }^{(6)}$.

Uma das primeiras medidas de controle dessas infecções foi a criação de Comissões de Controle de Infecção Hospitalar (CCIH), sob a recomendação da American Hospital Association, em 1958, com o

*Resumo da dissertação de mestrado apresentada à Área de Enfermagem Fundamental do Programa de Pós - Graduação do Departamento de Enfermagem Geral e Especializada da Escola de Enfermagem de Ribeirão Preto da Universidade de São Paulo (EERP-USP) 
objetivo de prover os hospitais americanos de um sistema que lhes permitisse apurar as causas das infecções neles adquiridas e dotá-los de instrumentos necessários contra possíveis ações legais movidas pela clientela. O entusiasmo pela vigilância epidemiológica das infecções hospitalares adentra as décadas seguintes com a implementação de novas medidas e empreendimentos, com vistas à prevenção e controle das mesmas.

No Brasil, a preocupação com o controle de infecções hospitalares surge na década de 60 através de publicações dos primeiros relatos sobre o tema. A primeira iniciativa para criação de uma $\mathrm{CCIH}$ data de 1963, no Hospital Ernesto Dornelles, em Porto Alegre- $\mathrm{RS}^{(7)}$. A criação de comissões multidisciplinares, vinculadas a hospitais universitários, surgiram a partir da década de 70. As próximas décadas foram contempladas com a publicação do Manual de Controle de Infecção Hospitalar ${ }^{(8)}$, pelo Ministério da Saúde, e com a promulgação de portarias, tornando obrigatória a instituição de CCIH em todos os hospitais brasileiros. Nessas portarias, são enfatizadas a composição das CCIH, suas atividades, as competências de seus membros, bem como algumas recomendações e indicadores epidemiológicos para o controle das infecções. ${ }^{(9,10,11)}$

Diferentes experiências passam a ser objeto de publicação, salientando a importância de implementação das CCIH e as dificuldades enfrentadas pelos profissionais com vistas a minimizar ou a buscar soluções para os problemas a elas relacionados..$^{(12 / 20)}$

As considerações contidas nos textos reforçam a tese de que para que as $\mathrm{CCIH}$ passem a desempenhar um papel de relevo no controle das infecções hospitalares, é necessário muito mais do que simplesmente criá-las burocraticamente, e que, para desempenharem um papel de relevo no controle das infecções, é fundamental o apoio político da administração do hospital. As instituições devem provê-las de condições mínimas essenciais para uma atuação efetiva, bem como de número adequado de recursos humanos, qualificados para o desenvolvimento de programas de prevenção e controle de IH.

Tendo em vista as considerações iniciais, propusemo-nos a desenvolver um estudo históricoorganizacional que retratasse a trajetória da $\mathrm{CCIH}$ do Hospital das Clínicas da Faculdade de Medicina de Ribeirão Preto - USP (HCFMRP-USP), desde a sua constituição até dezembro de 1997, com base na documentação disponível no referido hospital.

\section{METODOLOGIA}

Para a abordagem centralizada na $\mathrm{CCIH}$, utilizamos a metodologia de estudo de caso, com ênfase no Estudo Histórico - Organizacional, que se concentra em uma organização particular, durante um período, traçando seu desenvolvimento através da utilização de diferentes fontes de dados, inclusive de documentos escritos e submetidos à análise ${ }^{(21 / 24)}$.

Após confirmação de número suficiente de documentos arquivados pelo Serviço de Comunicações Administrativas do HCFMRP-USP, submetemos o projeto ao Comitê de Ética em Pesquisa do Hospital e ele mereceu aprovação, após consentimento do Presidente da CCIH, iniciamos a organização, em ordem cronológica, dos documentos disponíveis que se enquadravam em duas categorias, a saber: atas e processos.

As atas consistiam em registros escritos do desenvolvimento das reuniões da $\mathrm{CCIH}$, redigidas pela secretária da CCIH. Ao todo contamos com 351 atas, sendo a última, representativa da reunião de 9 de dezembro de 1997. Os processos caracterizavam-se como o conjunto de documentos compostos por ofícios, portarias, circulares, etc., relativos a um determinado assunto relacionado à CCIH. Nesse sentido, fizeram parte do nosso estudo um total de 22 processos desde 1978 até dezembro de 1997.

Os diferentes assuntos discutidos nas reuniões e registrados em atas, após várias leituras e releituras, foram classificados por diferenciação em cores e reagrupados em categorias e subcategorias sob um título genérico, em razão dos caracteres comuns a eles. Os processos foram inseridos nas apresentações das categorias e subcategorias, quando seu conteúdo apresentava associação à temática abordada em cada uma delas.

\section{RESULTADOS E COMENTÁRIOS}

\subsection{Histórico da CCIH: implantação, composi- ção e atribuições}

A iniciativa de criação da comissão foi determinada pela Portaria HCRP N ${ }^{\circ} 52$, de 26 de abril de 1973, quando o Superintendente do HCFMRP-USP, usando de suas atribuições, resolve criar a Comissão de Infecção Hospitalar do Hospital das Clínicas, subordinada diretamente à superintendência do hospital. Segundo a referida portaria, a comissão tinha como objetivo "assessorar a superintendência na elaboração 
de um programa de controle de infecções hospitalares, bem como exercer atividades para conhecimento contínuo da situação epidemiológica e das medidas profiláticas tomadas nos vários setores do hospital". Deveria ser constituída por representantes dos Departamentos: Clínico, Cirúrgico, de Medicina Social; Divisão de Enfermagem e de um microbiologista, devendo ser presidida por um de seus membros, designado pelo superintendente. A referida portaria especifica também as atribuições da comissão.

As reuniões dos membros da comissão deveriam ser realizadas, pelo menos, uma vez por mês e, extraordinariamente, quando necessário. No entanto, não foram localizadas as atas das reuniões até dezembro de 1978, quando foi divulgada nova portaria constituindo uma comissão especializada técnicoassistencial, designando outros membros na sua constituição. Vale considerar que, até 1978, o Hospital da Clínicas funcionava em um só prédio, hoje denominado Unidade de Emergência e que, a partir de 1979, passou a contar, também, com a Unidade do Campus Universitário. Com o Decreto $\mathrm{N}^{\circ} 13.297$, de março de 1979, a CCIH deveria ser composta por sete membros, sendo quatro docentes da FMRP-USP, dois médicos e um enfermeiro do Hospital das Clínicas, designados pelo Diretor Clínico, com aprovação do Conselho Deliberativo.

Somente a partir de abril de 1980, as reuniões realizadas quinzenalmente passaram a ser registradas em atas. Através dos registros, observamos que a composição da comissão variou através do tempo, em função de alterações entre seus membros.

Entre os membros executores da $\mathrm{CCIH}$, observamos que o enfermeiro sempre esteve presente durante o período investigado, chegando a ocupar até cinco representações. Seu número foi sendo reduzido, gradativamente, até a dois profissionais, como no final do período estudado. Em maio de 1989, foi contratado um médico infectologista com dedicação exclusiva junto à comissão, que passou a atuar também no controle de uso de antimicrobianos.

Outras comissões foram constituídas, tendo em vista a dimensão das atividades desenvolvidas pela CCIH. Dentre elas, podemos citar: Comissão Técnica de Vigilância Epidemiológica das Doenças de Notificação Compulsória, constituída em dezembro de 1989; Comissão de Uso e Controle de Antimicrobianos, em abril de 1993, e Comissão de Gerenciamento Interno dos Resíduos dos Serviços de Saúde, em março de 1996.

\subsection{Atividades desenvolvidas pela CCIH}

\subsubsection{Vigilância Epidemiológica das Infecções Hospitalares}

3.2.1.1 Atividades de coleta, análise e divulgação de dados dos casos de Infecções Hospitalares

A confecção de uma ficha de coleta de dados para o levantamento de infecções hospitalares, foi registrada em ata de abril de 1980. As primeiras unidades de internação investigadas foram as Clínicas Cirúrgica e Ortopédica do $\mathrm{HC}$, Campus. A partir dos resultados, foram levantados problemas relacionados à qualidade dos registros em prontuários e impressos do hospital e à falta de colaboração dos envolvidos para o fornecimento das informações, o que dificultava o levantamento das infecções hospitalares.

A coleta de dados passou a ser informatizada a partir de janeiro de 1983, através da Companhia de Processamento de Dados do Estado de São Paulo (PRODESP), sendo que a notificação dos casos de infecção hospitalar, constantes nas folhas de censo diário, ficava sob a responsabilidade das enfermeiras das Unidades de Internação. A alimentação do banco de dados ficava sob os cuidados das enfermeiras da comissão. A partir de um estudo realizado por membros da $\mathrm{CCIH}^{(25)}$, foi enfatizada a importância da busca ativa de casos de infecção hospitalar por enfermeiras fixas e treinadas, o que foi registrado em julho de 1988.

Os resultados das coletas de dados realizadas eram apresentados em reuniões da comissão, encaminhados à superintendência, ao conselho deliberativo e aos departamentos envolvidos. Em algumas referências, foram registrados seus encaminhamentos para o Ministério da Saúde e Centro de Vigilância Epidemiológica do Estado de São Paulo.

3.2.1.2. Atividades de vigilância de eventos e condições que afetam o risco de ocorrência de infecção hospitalar

Desde as primeiras reuniões registradas pela comissão, foi observada a preocupação com a descontaminação e desinfecção de ambientes e superfícies e a descontaminação, desinfecção e esterilização de artigos médico-hospitalares. Alguns dos assuntos foram registrados também em processos, como a vaporização de ambientes com desinfetantes, que deveria ser terminantemente abolida da rotina do hospital, segundo parecer da comissão. 
Outros assuntos discutidos e que merecem destaque foram os relacionados à desinfecção de banheiras, limpeza de pisos, incubadoras, berços, leitos, macas, cadeiras de roda, limpeza, descontaminação, desinfecção e esterilização de diferentes artigos e equipamentos médico-hospitalares e utilização de produtos químicos.

O controle de antimicrobianos foi outro assunto bastante discutido, com sugestões para a realização de levantamentos de consumo por clínica, implantação de uma ficha de controle de uso, culminando com a criação de uma Comissão de Uso e Controle de Antimicrobianos (CUCA), em abril de 1993.

O controle microbiológico da qualidade da água do hospital foi motivo de atenção constante da comissão, que buscou subsídios para a aquisição de novos equipamentos com vistas à tentativa de solucionar o problema.

As discussões conduzidas sobre os resíduos sólidos de serviços de saúde estiveram relacionadas ao sistema de acondicionamento, estocagem, incluindo perfurocortantes, ao tratamento dos resíduos, à incineração e coleta.

3.2.1.3 Atividades de avaliação de produtos químicos, recursos materiais e procedimentos

A preocupação com os produtos químicos foi desencadeada pela normatização estabelecida pelo Ministério da Saúde em junho de $1983^{(9)}$, quando a comissão encaminhou proposta ao Superintendente do HCFMRP-USP para que o hospital passasse a utilizar apenas produtos recomendados pela CCIH. A partir de 1984, a CCIH passou a realizar a análise de qualidade dos produtos químicos a serem adquiridos, com conseqüente elaboração de pareceres técnicos e auditorias, com vistas à definição dos mesmos. O trabalho conjunto com a Farmácia Industrial do hospital foi outra importante atividade de avaliação e padronização dos referidos produtos.

Dentre as atividades de avaliação de recursos materiais e procedimentos, destacam-se as relacionadas à lavagem das mãos, que permearam, praticamente, todo o período investigado. As discussões abordavam compra de saboneteiras para sabonete líquido, instalação de pias e de suportes para papel-toalha, envolvendo, inclusive, testes microbiológicos dos sabões líquidos.

O controle das soluções parenterais e conseqüente identificação de lotes contaminados culminou com a indicação de compra de sistema fechado de soro, em agosto de 1995.

Na mesma linha de raciocínio, algumas discussões foram localizadas em relação à prevenção de infecções urinárias através da utilização de sistema fechado de urina, critérios para sua reutilização, aquisição de sondas descartáveis e definição do controle de infecção urinária.

$\mathrm{O}$ isolamento de pacientes foi outro procedimento muito abordado nas reuniões da comissão, uma vez que esta ditava normas de isolamento e prestava assessoria, quando solicitada. Em 1988, foi aberto um processo destinado à construção de uma unidade especial para tratamento dos pacientes com aids, demonstrando a preocupação com o aumento do número de casos da síndrome e com a necessidade de uma área específica para o atendimento a esses pacientes, cuja construção foi concluída em julho de 1996. Todas as iniciativas no sentido de determinar condutas de isolamento contribuíram para a organização de um Manual de Isolamento, elaborado pela CCIH.

3.2.1.4. Atividades de controle de doenças de notificação compulsória e zoonoses

As doenças de notificação compulsória foram de responsabilidade da comissão no período de 1980 a 1989 , tendo sido discutidos problemas relacionados ao seguimento e encaminhamento dos casos de hanseníase, difteria, febre amarela, meningite meningocócica, neurocistecercose, sarampo e aids.

$\mathrm{O}$ controle de vetores também permeou várias discussões e esteve relacionado à presença de morcegos, pombos, ratos, moscas, mosquito transmissor da dengue, formigas e baratas no ambiente hospitalar, culminando com a sugestão de ser criada uma equipe de servidores encarregada de realizar tal controle.

\subsubsection{Atividades de ensino e educação conti- nuada}

As atividades de ensino e educação continuada constituíram um importante papel da comissão. Elas estiveram relacionadas à orientação dos servidores do hospital, alunos, residentes e professores e treinamento dos mesmos para atuarem na prevenção e controle da infecção hospitalar junto a outras $\mathrm{CCIH}$, à divulgação de eventos, palestras, artigos, teses e livros relacionados à Infecção Hospitalar. A qualificação e especialização dos membros executivos da comissão, foram sempre tema de importância, bem como da participação destes em eventos, palestras e cursos em outros hospitais e universidades. A comissão ofere- 
ceu campo de estágio a vários profissionais e assessoria a outros serviços. O papel educativo das enfermeiras da comissão esteve muito evidente. Em 1985, o HCFMRP-USP foi credenciado pelo Ministério da Saúde como Centro de Treinamento sobre Infecção Hospitalar, e passou a promover diversos cursos para profissionais de saúde, envolvidos com o controle de infecção hospitalar nos hospitais da região de Ribeirão Preto. A comissão participou da elaboração de diversos manuais informativos, que foram distribuídos no hospital, com os temas de isolamento de pacientes, precauções e coleta microbiológica, e de algumas campanhas educativas. Vale ressaltar a preocupação com a criação de uma disciplina sobre infecção hospitalar para a graduação da FMRP-USP, que foi oferecida, pela primeira vez, em 1995.

\subsubsection{Atividades de assessoria em projetos re- lacionados à planta física}

A assessoria prestada a projetos de planta física do hospital esteve relacionada às reformas realizadas nas unidades do Campus e UE. No entanto, tal assessoria não se deu de forma contínua, considerando-se que, em algumas situações, não era solicitado o parecer da comissão que, assim mesmo, procurava manter contato com os locais submetidos à reforma.

\subsubsection{Atividades relacionadas à segurança do trabalho}

Os riscos biológicos e sua prevenção sempre foram alvo de discussão da CCIH. Esses problemas, geralmente, eram trazidos por representantes do Serviço de Assistência Médica e Social do Pessoal (SAMSP) e Serviço Especializado de Segurança e Medicina do Trabalho (SESMT). Os acidentes com materiais perfurocortantes, por reencape de agulhas e inadequação no acondicionamento, desencadeou a elaboração de protocolos de orientação de condutas. Outros procedimentos que foram avaliados pela comissão foram o transporte de roupas sujas, materiais biológicos e cadáveres, com recomendações sobre a necessidade de utilização de equipamentos de proteção individual e confecção de suportes para transporte.

\section{CONSIDERAÇÕES FINAIS}

A opção em realizar um estudo históricoorganizacional da trajetória da CCIH do HCFMRPUSP, desde a sua constituição, em 1973, até 1997, com base na documentação arquivada junto ao Serviço de
Comunicações Administrativas do Hospital das Clínicas, colocou-nos diante do desafio de selecionar, dentre tantos documentos, aqueles que nos levassem a retratar, cronologicamente o conjunto de atividades desenvolvidas pela referida comissão, durante o citado período, o que foi possível depois de repetidas leituras e muita objetividade na análise dos documentos.

Na descrição dos fatos relacionados à implantação, composição e atribuições da comissão, percebemos as alterações e as conquistas advindas de um trabalho desenvolvido com determinação e empenho por parte dos participantes, na manutenção de um único objetivo: assessorar a Superintendência do HC na condução e manutenção de um programa de controle de infecção hospitalar, através da execução de atividades para a sua prevenção e controle, trabalho que, apesar de seu caráter de assessoria, levou a comissão a ser reconhecida pela Comunidade. No final do período estudado, a CCIH mantinha-se em ação, ainda que com número insuficiente de membros efetivos, principalmente aqueles relacionados à enfermagem, que é a responsável pela realização do trabalho executivo de uma CCIH.

As conquistas advindas do desenvolvimento das atividades de ensino e educação continuada apontaram para o potencial de que dispõe uma comissão compromissada em desenvolver um trabalho com qualidade. Tais atividades foram direcionadas para duas vertentes: a interna e a externa, ou seja, para o aprimoramento dos próprios servidores através de orientações, treinamentos, elaboração de manuais informativos e campanhas, e para o atendimento de solicitações de outras instituições preocupadas com o problema das infecções hospitalares e com a organização de uma estrutura voltada para o seu controle.

Por último, queremos registrar que a experiência em conduzir um estudo desta natureza nos fez refletir constantemente sobre o desafio de realizar uma análise histórica com base em documentos disponíveis. Apesar da tentativa de assegurar a fidedignidade dos fatos analisados, não podemos deixar de considerar que o trabalho da comissão, como um todo, extrapola o que foi registrado, considerando-se as possíveis deficiências da comunicação escrita.

Registramos, também, que nossa experiência com o assunto estudado contribuiu para fortalecer o respeito e o reconhecimento a um grupo de profissionais que enfrenta o desafio de buscar alternativas de prevenção e controle das $\mathrm{IH}$, através da conscientiza- 
ção de todos os envolvidos com a mesma, e para a compreensão da dinâmica das reuniões e das atividades desenvolvidas por tal grupo.

Refletir, no hoje, os problemas enfrentados no ontem e alertar para os problemas do amanhã, eis o saldo positivo, ao concretizarmos este estudo histórico da Comissão de Controle de Infecção Hospitalar do HCFMRP-USP.

SILVA MFI \& OLIVEIRA SANTOS BM.. Historical-organizational study about the hospital infection control committee of an university hospital. Medicina, Ribeirão Preto 34: 170-176, april/june 2001.

ABSTRACT: This work is characterized by a historical - organizational study about the Hospital Infection Control Committee trajectory at Hospital das Clínicas from Faculdade de Medicina de Ribeirão Preto da Universidade de São Paulo, since its constitution (1973) until this moment (1997), based on registers and processes organized chronologically. The methodology used for this approach was the case study, characterized by detailed examination of documents, with the purpose of determining categories and sub - categories that had been representative of activities complex developed by the Committee. The activities developed by the Committee were centered basically on infection epidemiological vigilance, continued teaching and education, project advising in relation to physical plans and work safety. This work can also contribute as an easy to reach source of information for the Committee itself, for constitution of other Committees and even though to future researches.

UNITERMOS: Commissions. Infection Control. Nosocomial Infection. History.

\section{REFERÊNCIAS BIBLIOGRÁFICAS}

1 - ZANON U; AGUIAR N \& COSTA BG. Diretrizes para a organização da comissão de controle de infecções hospitalares In: ZANON U \& NEVES J. Infecções hospitalares: prevenção, diagnóstico e tratamento. Medsi, Rio de Janeiro, p. 47-55, 1987.

2 - LA FORCE FM. The control of infections in hospitals: 1750 to 1950. In: WENZEL RP. Prevention and control of nosocomial infection. 2nd.ed., Williams \& Wilkins, Baltimore, p.112, 1993.

3 - STARLING CEF \& TAVARES AP. Aspectos históricos do controle das infecções hospitalares. In: STARLING CEF; PINHEIRO SMC \& COUTO BRGM. Vigilância epidemiológica das infecções hospitalares na prática diária. Cuatiara, Belo Horizonte, p. 1 - 23, 1993.

4 - COUTO CR \& NOGUEIRA JM. História do controle de infecção hospitalar e a situação brasileira. In: COUTO CR; PEDROSA TMG \& NOGUEIRA JM. Infecção hospitalar: epidemiologia e controle. Medsi, Rio de Janeiro, p. 1-4, 1997.

5 - RODRIGUES EAC. Histórico das infecções hospitalares. In: RODRIGUES, E.A.C. ; MENDONÇAJS; AMARANTE JMB; ALVES FILHO MB; GRINBAUM RS \& RICHTMANN R. Infecções hospitalares: prevenção e controle. Sarvier, São Paulo, p.3 -27, 1997.

6 - BRASIL. Ministério da Saúde. Portaria N․ํ 2.616 de 12 de maio de 1998. Resolve expedir, na forma dos anexos I, II, III, IV e $\mathrm{V}$, diretrizes e normas para a prevenção e o controle das infecções hospitalares, ficando revogada a Portaria n. 0930. Diário Oficial da União, Brasília, Seção 1, p.133 - 5, 13 maio, 1998.
7 - PEREIRA MS. Infecção hospitalar no Brasil: um enfoque sobre o seu controle. Dissertação de Mestrado. Escola de Enfermagem de Ribeirão Preto da USP, Ribeirão Preto, p.1123,1987 .

8 - BRASIL. Ministério da Saúde. Secretaria Nacional de Organização E Desenvolvimento de Serviços de Saúde. Manual de controle de infecção hospitalar. Brasília, 1987.

9 - BRASIL. Ministério da Saúde. Portaria N. 196 de 24 de junho de 1983. Dispõe sobre as normas técnicas sobre a prevenção de infecções hospitalares. Diário Oficial da União, Brasília, Seção 1, p.11.319-23, 28 jun.,1983.

10 - BRASIL. Ministério da Saúde. Portaria N․ำ 930 de 27 de agosto de 1992. Estabelece normas para o controle da infecção hospitalar e revoga a Portaria n.196. Diário Oficial da União, Brasília, Seção 1, p.12.279 - 81, 4 set. ,1992.

11 - BRASIL. Ministério da Saúde. Lei n. 9.431 de 6 de janeiro de 1997. Dispõe sobre a obrigatoriedade da manutenção de programa de controle de infecções hospitalares pelos hospitais do Pais. Diário Oficial da União, Brasília, Seção 1, p. 265, 7 jan., 1997.

12 - ÉVORAYDM \& ALMEIDA ECS. Comissão de controle de infecção hospitalar de um hospital - escola. Rev Paul Hosp 31: 70-76, 1983.

13 - ROCHA MLQ. Atuação dos enfermeiros nas comissões de controle de infecção hospitalar - relato de experiência. Rev Paul Enf 5:151 - 154, 1985.

14 - SILVA CM Atuação da enfermeira na comissão de controle de infecção hospitalar. Rev Paul Enf 5: 154 - 158, 1985. 
15 - PEREIRA MS \& MORIYA TM. Infecção hospitalar: Estrutura básica de vigilância e controle. AB, Goiânia, 1994.

16 - CAVALCANTE MDA \& COSTA MP. O controle da infecção hospitalar no Hospital de Base do Distrito Federal de Brasília, 1984-1986. HFA Publ Téc Cient 20: 389-98, 1988.

17 - PRADE SS. Método de controle das infecções hospitalares orientado por problemas. Atheneu, São Paulo, 1988.

18 - LACERDA RA Atividades das enfermeiras das comissões de controle de infecção hospitalar de hospitais do município de São Paulo. Rev Esc Enf USP 23: 79-89, 1989.

19 - CUNHA FLA. Implantação da comissão de controle de infecção hospitalar em Hospital de Área. Rev Med Aeronaut Bras 39: 29-35, 1989.

20 - PEREIRA MS; NAKATANI AYK \& SOUZA ACS. Organização técnica e funcional dos serviços de controle de infecção hospitalar existentes nos hospitais do município de Goiânia - Goiás. Rev Bras Enf 47: 231- 240, 1994.
21 - BOGDAN RC; BIRTEN SK. Qualitative research for education: an introduction for to theory and methods., Allys and Bacon, Boston,1982.

22 - POLIT DF \& HUNGLER BPH. Nursing research: principles and methods. 2.ed, J.P. Lippincott, Philadelphia, 1983.

23 - LUDKE M. Pesquisa em educação: abordagens qualitativas. EPU, São Paulo, 1986.

24 - BARDIN L. Análise de conteúdo. Tradução de Luís Antero Reto e Augusto Pinheiro, Edições 70, São Paulo, 1977.

25 - HAYASHIDA M; FURLAN MLS; DEL LAMA J \& COSTA JC. Avaliação do sistema de notificação de infecção hospitalar do HCFMRP-USP Campus. Comissão de Controle de Infecção Hospitalar do HCFMRP-USP, Ribeirão Preto, 1986./Datilografado/.

Recebid para publicação em 06/09/2000

Aprovado para publicação em 26/06/2001 